\title{
REVIEW ON QCD SUM RULES CALCULATIONS FOR EXOTICS CHARMONIUM
}

\author{
MARINA NIELSEN \\ Instituto de Física, Universidade de São Paulo, \\ Caixa Postal 66318, 05389-970 São Paulo, SP, Brazil \\ mnielsen@if.usp.br
}

\begin{abstract}
Many new states in the charmonium mass region were recently discovered by the BaBar, Belle and CDF Collaborations. We use the QCD Sum Rule approach to study the possible structure of some of these states.

Keywords: QCD sum rules; exotic states.

PACS numbers: 12.38.Lg, 12.40.Yx, 14.40.Lb, 14.40.Rt
\end{abstract}

\section{Introduction}

Most of the new charmonium states discovered in recent years in the mass region around $4000 \mathrm{MeV}$ is due to the work of the Belle and BaBar Collaborations at the $B$ factories. Many of these new states, called $X, Y, Z$ particles, do not seem to have a simple $c \bar{c}$ structure. Although the masses of these states are above the corresponding thresholds of decays into a pair of open charm mesons, they decay into $J / \psi$ or $\psi^{\prime}$ plus pions, which is unusual for $c \bar{c}$ states. Besides, their masses and decay modes are not in agreement with the predictions of potential models, which, in general, describe very well $c \bar{c}$ states. For these reasons they are considered as candidates for exotic states, with a more complex structure than the simple quark-antiquark state, like hybrid, molecular or tetraquark states. The idea of unconventional quark structures is quite old and the light scalar mesons were the first candidates for tetraquark exotic states. However, despite decades of progress, no exotic meson has been conclusively identified. These states are allowed by the strong interactions, both at the fundamental level and at the effective level, and their absence in the experimentally measured spectrum has always been a mystery. Here we discuss some of these new states using the QCD sum rules (QCDSR) approach.

\section{QCD Sum Rules}

The method of the QCDSR was first introduced by Shifman, Vainshtein and Zakharov $^{1}$ and applied to the mesons. They demonstrated that, for the determination of the mass of the state using the method, the non-perturbative power 
corrections are more important than the strong coupling, $\alpha_{s}$, corrections. The nonperturbative power corrections were introduced through a series expansion of operators. As the dimension of the operators increase, the power of the momentum transfer, $Q^{2}$, in the denominator of the terms also increases, giving a series in $1 / Q^{2}$ which can be truncated for large values of $Q^{2}$. The sum rule method was latter extended to baryons by Ioffe ${ }^{2}$ and Chung et al. ${ }^{3}$. Since then the QCDSR technique has been applied to study numerous hadronic properties with various flavor content and is discussed in many reviews ${ }^{4,5}$ emphasizing different aspects of the method.

The method is based in the evaluation of the correlation function:

$$
\Pi(q) \equiv i \int d^{4} x e^{i q \cdot x}\left\langle 0\left|T\left[j(x) j^{\dagger}(0)\right]\right| 0\right\rangle
$$

in two different ways. At the quark level in terms of quark and gluon fields, and at the hadronic level by introducing hadron parameters. In Eq. (1) $j(x)$ is a current which has the quantum numbers of the hadron we want to study.

In what follows we present some results of the QCDSR calculations on the new charmonium states presented in Table 1.

Table 1. Charmonium states observed in the last years.

\begin{tabular}{|c|c|c|c|}
\hline state & production mode & decay mode & ref. \\
\hline$X(3872)$ & $B \rightarrow K X(3872)$ & $J / \psi \pi \pi$ & 6 \\
$Z_{1}^{+}(4050)$ & $B^{0} \rightarrow K^{-} Z_{1}^{+}(4050)$ & $\chi_{c 1} \pi^{+}$ & 7 \\
$Z_{2}^{+}(4250)$ & $B^{0} \rightarrow K^{-} Z_{1}^{+}(4250)$ & $\chi_{c 1} \pi^{+}$ & 7 \\
$Y(4260)$ & $e^{+} e^{-} \rightarrow \gamma_{I S R} Y(4260)$ & $J / \psi \pi \pi$ & 8 \\
$Y(4360)$ & $e^{+} e^{-} \rightarrow \gamma_{I S R} Y(4260)$ & $\psi^{\prime} \pi \pi$ & 9 \\
$Z^{+}(4430)$ & $B^{0} \rightarrow K^{-} Z^{+}(4430)$ & $\psi^{\prime} \pi^{+}$ & 10 \\
$Y(4660)$ & $e^{+} e^{-} \rightarrow \gamma_{I S R} Y(4660)$ & $\psi^{\prime} \pi \pi$ & 9 \\
\hline
\end{tabular}

We assume these states to be composed by four quarks either in a molecular or in a tetraquark configuration. For more details we refer the reader to our recent review on the subject ${ }^{11}$.

\section{3. $X, Y$ and $Z$ charmonium states}

\section{1. $X(3872)$}

The $X(3872)$ was the first non-conventional charmonium, i.e., it has a mass significantly smaller than the one predicted by the standard quark model with these quantum numbers, which are most probably $J^{P C}=1^{++}$. Moreover, the $X$ decays with comparable strength into $J / \psi$ plus two and $J / \psi$ plus three pions, showing a strong isospin violation which is not compatible with a $c-\bar{c}$ state. Finally, this state has a decay width of less than $2.3 \mathrm{MeV}$, which is too small to be easily accounted for. 
If we assume the $X$ to be described by a $J^{P C}=1^{++}$four-quark current either in a diquark-antidiquark configuration:

$$
j_{\mu}^{(q, d i)}=\frac{i \epsilon_{a b c} \epsilon_{d e c}}{\sqrt{2}}\left[\left(q_{a}^{T} C \gamma_{5} c_{b}\right)\left(\bar{q}_{d} \gamma_{\mu} C \bar{c}_{e}^{T}\right)+\left(q_{a}^{T} C \gamma_{\mu} c_{b}\right)\left(\bar{q}_{d} \gamma_{5} C \bar{c}_{e}^{T}\right)\right]
$$

or in a molecular $D \bar{D}^{*}$ configuration:

$$
j_{\mu}^{(q, m o l)}(x)=\frac{1}{\sqrt{2}}\left[\left(\bar{q}_{a}(x) \gamma_{5} c_{a}(x) \bar{c}_{b}(x) \gamma_{\mu} q_{b}(x)\right)-\left(\bar{q}_{a}(x) \gamma_{\mu} c_{a}(x) \bar{c}_{b}(x) \gamma_{5} q_{b}(x)\right)\right],
$$

we find that it is possible to describe its mass ${ }^{12,13}$, but it is not possible to describe its width ${ }^{14}$. The mass obtained using the current in Eq. (2) was $M_{X}=(3.92 \pm$ 0.13) $\mathrm{GeV}^{12}$. In the case of the current Eq. (3), the result for the mass obtained in ref. ${ }^{13}$ was $M_{X}=(3.87 \pm 0.07) \mathrm{GeV}$, both in good agreement with the experimental mass. However, the decay width obtained in ref. ${ }^{14}$ for the decay mode $X \rightarrow J / \psi \pi \pi$ was $\Gamma_{X \rightarrow J / \psi \pi \pi}=(50 \pm 15) \mathrm{MeV}$, much bigger than the experimental upper limit. Therefore, from a QCDSR calculation it is not possible to explain the small width of the $X(3872)$ if it is a pure four-quark state. In ref. ${ }^{15}$ the $X(3872)$ was treated as a mixture of a $c \bar{c}$ state with a molecular state:

$$
J_{\mu}^{q}(x)=\sin (\alpha) j_{\mu}^{(q, m o l)}(x)+\cos (\alpha) j_{\mu}^{(q, 2)}(x),
$$

with $j_{\mu}^{(q, m o l)}(x)$ given in Eq. (3). From the results presented in ref. ${ }^{15}$ one can conclude that it is possible to reproduce the experimental mass of the $X(3872)$ for a wide range of mixing angle, $\alpha$, but, as observed $i^{15}$, it is not so easy to reproduce the experimental decay width. In ref. ${ }^{15}$ it was shown that with a mixing angle $\alpha=9^{0} \pm 4^{0}$ in Eq. (4) it is possible to describe the experimental mass of the $X(3872)$ with a decay width of $\Gamma(X \rightarrow J / \psi(n \pi))=(9.3 \pm 6.9) \mathrm{MeV}$, which is compatible with the experimental upper limit.

To summarize, we could say that, in QCDSR it is possible to satisfactorily explain all the $X(3972)$ features assuming that it is a mixture of a $c-\bar{c}$ component and with a meson molecule component. This molecular component, in its turn, must be a mixture of $D^{0} D^{* 0}$ and $D^{+} D^{*-}$ components $^{15}$.

\section{2. $Y\left(J^{P C}=1^{--}\right)$states}

The masses and widths of the three $Y\left(J^{P C}=1^{--}\right)$states: $Y(4260), Y(4360)$ and $Y(4660)$ are not consistent with any of the established $1^{--}$charmonium states ${ }^{16}$, and they can also be candidates for multiquark states or charmonium hybrids.

A possible interpolating operator representing a $J^{P C}=1^{--}$tetraquark state with symmetric spin distribution can be made with the following diquark blocks: $[c s]_{S=0}[\bar{c} \bar{s}]_{S=1}+[c s]_{S=1}[\bar{c} \bar{s}]_{S=0}$. With a current made with these diquarks, the mass found in ref. ${ }^{17}$ was $m_{Y}=(4.65 \pm 0.10) \mathrm{GeV}$ in excellent agreement with the mass of the $Y(4660)$ meson. Replacing the strange quarks by a generic light quark $q$ in the current, we obtained ${ }^{17}$ the mass $m_{Y}=(4.49 \pm 0.11) \mathrm{GeV}$ for the $1^{--}$state represented by the symmetric spin distribution: $[c q]_{S=0}[\bar{c} \bar{q}]_{S=1}+[c q]_{S=1}[\bar{c} \bar{q}]_{S=0}$. This 
value is bigger than the $Y(4360)$ mass, but it is consistent with it considering the uncertainty.

The $Y$ mesons can also be described by molecular type currents. A current with $J^{P C}=1^{--}$and a symmetrical combination between scalar and vector mesons is given by $j_{\mu}=\frac{1}{\sqrt{2}}\left[\left(\bar{s}_{a} \gamma_{\mu} c_{a}\right)\left(\bar{c}_{b} s_{b}\right)+\left(\bar{c}_{a} \gamma_{\mu} s_{a}\right)\left(\bar{s}_{b} c_{b}\right)\right]$. The mass obtained in ref. ${ }^{17}$ for this current was $m_{D_{s 0} \bar{D}_{s}^{*}}=(4.42 \pm 0.10) \mathrm{GeV}$, which is more in agreement with the $Y(4350)$ mass than with the $Y(4660)$ one.

To consider a molecular $D_{0} \bar{D}^{*}$ current with $J^{P C}=1^{--}$, one has only to change the strange quarks in the current by a generic light quark $q$. The mass obtained with such current was $^{17} m_{D_{0} \bar{D}^{*}}=(4.27 \pm 0.10) \mathrm{GeV}$, in excellent agreement with the mass of the meson $Y(4260)$. From the measured spectra, it seems that the $Y(4260)$ is consistent with a non-strange molecular state $D_{0} \bar{D}^{*}$.

A $J^{P C}=1^{--}$molecular current can also be constructed with pseudoscalar and axial-vector mesons. A molecular $D \bar{D}_{1}$ current was used in ref. ${ }^{18}$. The mass obtained with this current was $m_{D \bar{D}_{1}}=(4.19 \pm 0.22) \mathrm{GeV}$. Therefore, considering the errors, the molecular $D \bar{D}_{1}$ assignment for the meson $Y(4260)$ is also possible, in agreement with the findings of ref. ${ }^{19}$, where a meson exchange model was used to study this new meson.

The $Y(4260)$ can also be interpreted as a $J / \psi f_{0}(980)$ molecular state ${ }^{20}$.

\section{3. $Z^{+}$states}

The $Z^{+}(4430), Z_{1}^{+}(4050)$ and $Z_{2}^{+}(4250)$ were observed by Belle Collaboration. Since the minimal quark content of these states is $c \bar{c} u \bar{d}$, they are prime candidates for a multiquark mesons.

In ref. ${ }^{21}$ QCD sum rules were used to study the $Z^{+}(4430)$ considered as a $D^{*} D_{1}$ molecular state with $I^{G} J^{P}=1^{+} 0^{-}$. The mass obtained was $M_{Z^{+}}=$ $(4.40 \pm 0.10) \mathrm{GeV}$ in an excelent agreement with the experimental mass. To check if the $Z^{+}(4430)$ could also be described as a diquark-antidiquark state, in ref. ${ }^{22}$ different currents were considered with $J^{P}=0^{-}$and $1^{-}$. The results obtained were: $M_{Z}\left(0^{-}\right)=(4.52 \pm 0.09) \mathrm{GeV}$ and $M_{Z}\left(1^{-}\right)=(4.84 \pm 0.14) \mathrm{GeV}$. From these results we conclude that while it is also possible to describe the $Z^{+}(4430)$ as a diquark-antidiquark state with $J^{P}=0^{-}$, the $J^{P}=1^{-}$configuration is disfavored.

Due to the closeness of the $Z_{1}^{+}(4050)$ and $Z_{2}^{+}(4250)$ masses to the $D^{*} \bar{D}^{*}(4020)$ and $D_{1} \bar{D}(4285)$ thresholds, these states could also be interpreted as molecular states. The QCD sum rules formalism was used to study the $D^{*} \bar{D}^{*}$ and $D_{1} \bar{D}$ molecular states with $I^{G} J^{P}=1^{-} 0^{+}$and $1^{-} 1^{-}$respectively. The mass obtained for these molecular states are: $M_{D^{*} D^{*}}=(4.15 \pm 0.12) \mathrm{GeV}$, and $M_{D_{1} D}=(4.19 \pm 0.22) \mathrm{GeV}$. In ref. ${ }^{23}$ it was found that the inclusion of the width, in the phenomenological side of the sum rule, increases the obtained mass for molecular states. This means that the introduction of the width in the sum rule calculation, increases the mass of the $D^{*} \bar{D}^{*}$ and $D_{1} \bar{D}$ molecules. As a result, the mass of the $D_{1} \bar{D}$ molecule will be closer to the observed $Z_{2}^{+}(4250)$ mass, and the mass of the $D^{*} \bar{D}^{*}$ molecule will be far 
from the $Z_{1}^{+}$(4050) mass. Therefore, the authors of ref. ${ }^{23}$ conclude that it is possible to describe the $Z_{2}^{+}(4250)$ resonance structure as a $D_{1} \bar{D}$ molecular state with $I^{G} J^{P}=1^{-} 1^{-}$quantum numbers, and that the $D^{*} \bar{D}^{*}$ state is probably a virtual state that is not related with the $Z_{1}^{+}(4050)$ resonance-like structure.

\section{Conclusions}

We have computed the masses of some $X, Y$ and $Z$ states, recently observed by BaBar and Belle Collaborations, using the QCDSR approach. In some cases a tetraquark configuration was favored, as the $Y(4660)$, and in some other cases a molecular configuration was favored. like the $Y(4260)$ and $Z^{+}(4430)$. In the case of $X(3872)$ we found that it is only possible to explain all the available experimental data if it is a mixed state with charmonium and molecular $D \bar{D}^{*}$ components.

\section{Acknowledgments}

This work was supported by the brazilian funding agencies FAPESP and CNPq.

\section{References}

1. M. A. Shifman, A. I. Vainshtein, V. I. Zakharov, Nucl. Phys. B147, 385 (1979); 147, $448 ; \mathbf{1 4 7}, 519$.

2. B. L. Ioffe, Nucl. Phys. B188, 317 (1981); 191, 591(E).

3. Y. Chung, H. G. Dosch, M. Kremer, D. Schall, Phys. Lett. B102, 175 (1981); Nucl. Phys. B197, 55 (1982).

4. L.J. Reinders, H. Rubinstein and S. Yazaki, Phys. Rep. 127, 1 (1985).

5. S. Narison, QCD as a theory of hadrons, Cambridge Monogr. Part. Phys. Nucl. Phys. Cosmol. 17, 1 (2002); QCD spectral sum rules, World Sci. Lect. Notes Phys., 26, 1 (1989); Acta Phys. Pol. 26, 687 (1995); Riv. Nuov. Cim.10N2, 1 (1987); Phys. Rept., 84, 263 (1982).

6. Belle Collab. (S.-K. Choi et al.) Phys. Rev. Lett. 91, 262001 (2003).

7. Belle Collab. (R. Mizuk et al.) Phys. Rev. D78, 072004 (2008).

8. BaBar Collab. (B. Aubert et al.) Phys. Rev. Lett. 95, 142001 (2005).

9. Belle Collab. (X.L. Wang et al.) Phys. Rev. Lett. 99, 142002 (2007).

10. Belle Collab. (K. Abe et al.) Phys. Rev. Lett. 100, 142001 (2008).

11. M. Nielsen, F. S. Navarra and S. H. Lee, arXiv:0911.1958, to appear in Phys. Rep.

12. R.D. Matheus et al., Phys. Rev. D 75, 014005 (2007).

13. S.H. Lee, M. Nielsen and U. Wiedner, Jour. Korean Phys. Soc. 55, 424 (2009).

14. F.S. Navarra, M. Nielsen, Phys. Lett. B 639, 272 (2006).

15. R.D. Matheus et al., Phys. Rev. D 80, 056002 (2009).

16. S.-L. Zhu, Int. J. Mod. Phys. E 17, 283 (2008).

17. R.M. Albuquerque and M. Nielsen, Nucl. Phys. A 815, 53 (2009).

18. S.H. Lee, K. Morita, M. Nielsen, Nucl. Phys. A 815, 29 (2009).

19. G.-J. Ding, Phys. Rev. D 79, 014001 (2009).

20. A.M. Torres et al., Phys.Rev. D 80, 094012 (2009).

21. S.H. Lee, A. Mihara, F.S. Navarra and M. Nielsen, Phys. Lett. B661, 28 (2008).

22. M.E. Bracco et al., Phys. Lett. B671, 240 (2009).

23. S.H. Lee, K. Morita and M. Nielsen, Phys. Rev. D 78, 076001 (2008). 